BMJ Open

Sport \&

Exercise

Medicine

\section{Exercise time cues (zeitgebers) for human circadian systems can foster health and improve performance: a systematic review}

To cite: Lewis P, Korf HW, Kuffer $\mathrm{L}$, et al. Exercise time cues (zeitgebers) for human circadian systems can foster health and improve performance: a systematic review. BMJ Open Sport \& Exercise Medicine 2018:4:e000443. doi:10.1136/ bmjsem-2018-000443

Accepted 12 November 2018

\section{Check for updates}

(c) Author(s) (or their employer(s)) 2018. Re-use permitted under CC BY-NC. No commercial re-use. See rights and permissions. Published by BMJ.

${ }^{1}$ Institute and Policlinic for Occupational Medicine, Environmental Medicine and Prevention Research, University Hospital of Cologne, Cologne, Germany

${ }^{2}$ Institute of Anatomy I, University of Düsseldorf, Düsseldorf, Germany

Correspondence to Dr Philip Lewis, philip.lewis@ uk-koeln.de

\section{ABSTRACT \\ Background Circadian system time cues (zeitgebers) acting synergistically at the right times can foster chronobiological homeostasis and ultimately health. Modern 24/7 societies are challenging chronobiological homeostasis and public health. Exercise has been discussed as a potential zeitgeber for the human circadian system. Thus, if timed correctly, exercise may help in maintenance of chronobiological homeostasis and foster public health amidst increasingly challenging 24/7 lifestyles.}

Objective To test, using a systematic review of the literature, the following hypothesis: exercise is a zeitgeber for the human circadian system.

Data sources The PubMed database was systematically searched on 19 0ctober 2017 for relevant scientific studies and reports concerning chronobiology and exercise. Eligibility criteria were defined to include articles considering exercise as a potential zeitgeber for human circadian rhythmicity or chronobiological effects of exercise on health and/or physical performance. Cognitive effects and effects on children were excluded from the synthesis.

Results Our systematic literature search and synthesis is compatible with the validity of the hypothesis. We report that potential exercise-zeitgeber properties may be used to improve health and performance.

Conclusions Informed timing of exercise, specific to the circadian rhythm phase and zeitgeber exposure of the individual, must be advocated in performance and disease contexts as an adjunct therapeutic or preventative strategy and physical enhancer.

\section{INTRODUCTION}

In the 1950s, Jürgen Aschoff, a nestor of chronobiology, suggested the term zeitgeber (time cue, from German $)^{1}$ and operationalised its concept $^{2}$ as an external signal that synchronises physiological 24-hour periodicity. We know of myriad, day-periodic, fundamental physiological processes in humans that are governed by an endogenous clock-like system that is receptive to zeitgebers such as the pivotal light-dark cycle. This system is known as the circadian system which generates
Key messages

Modern societal demands such as shiftwork, transmeridian travel, light-at-night, light emitting devices and 24/7 lifestyles challenge the human circadian system.

- Exercise, acting as a zeitgeber (time cue) for the circadian system, may be used to foster chronobiological homeostasis and ultimately health.

- We systematically reviewed the literature to test whether exercise can be a zeitgeber for the human circadian system-the supporting evidence is strong.

- Informed timing of exercise can be advocated in performance and disease contexts as a physical enhancer or as an adjunct therapeutic or preventative strategy.

rhythms of approximately 24-hour period length ('circadian' from Latin circa and dies, meaning 'about a day' ${ }^{3}$ ).

The 'master clock' or 'pacemaker', that receives light-dark information via the retinohypothalamic tract from intrinsically photosensitive retinal ganglion cells, is located in the suprachiasmatic nuclei (SCN) in the anterior hypothalamus region of the brain. ${ }^{4}$ Neuroendocrine output is the current postulated mechanism for circadian alignment throughout the body. ${ }^{5}$ Importantly, the synchronisation (or entrainment) of circadian rhythms to zeitgeber periods significantly contributes to physical performance, cognitive performance and overall health. ${ }^{7-9}$

Zeitgeber information which an individual experiences can come from multiple sources and may interact with extreme consequences. It may allow said individual to 'run-like clockwork' or may turn his/her circadian system into a 'ticking time bomb'. ${ }^{9}$ Modern societal demands can provide conflicting zeitgeber information insufficient for, or detrimental to, the chronobiological health of individuals and populations. For example, shiftwork that involves circadian disruption was classified 
Table 1 Search string and inclusion/exclusion criteria as of 19 October 2017

Search string (“Chronobiology Discipline"[Mesh] OR "Circadian Clocks"[Mesh] OR "Circadian Rhythm”[Mesh] OR "Sleep Phase Chronotherapy"[Mesh] OR "Biological Clocks"[Mesh] OR "Jet Lag Syndrome"[Mesh] OR chrono* OR circadian OR morningness OR eveningness OR jet-lag OR time zone OR zeitgeber OR synchronizer OR "entraining agent") AND ("Sports"[Mesh] OR "Sports Medicine"[Mesh] OR "Athletic Performance"[Mesh] OR "Task Performance and Analysis"[Mesh] OR "Resistance Training"[Mesh] OR "High-Intensity Interval Training"[Mesh] OR "Circuit-Based Exercise"[Mesh] OR "Exercise"[Mesh] OR "Plyometric Exercise"[Mesh] OR "Athletes"[Mesh] OR "Exercise Tolerance"[Mesh] OR sport* OR train* OR athletic OR athlete* OR exercise) AND (performance OR health)

Inclusion criteria Original articles that must consider effects of exercise or physical activity as a potential zeitgeber on circadian rhythmicity, performance, or health in humans and be in English.

Exclusion criteria The effects of exercise on cognitive states are well documented; thus, studies on mood, subjective exertion, non-sport-associated cognitive ability, and homeostatic drive to sleep were excluded. Furthermore, studies on children and early adolescent teenagers were excluded, as were studies of shiftworkers or airline crew. The challenging $24 / 7$ society differentially affects adults, who are the scope of this work, and the impact of shift-work or airline work may allow zeitgeber interactions with exercise that we cannot account for.

as a group 2A carcinogen ("probably carcinogenic to humans") by the International Agency for Research on Cancer in 2010. ${ }^{10}$ Plausible effects of modern societal demands and conflicting zeitgebers could contribute to the current epidemic of sleep deficiency and the increasing global burden of mental, metabolic, cardiovascular and cancer diseases associated with chronobiological ill-health. ${ }^{9-17}$ Conversely, zeitgeber information acting synergistically at the right times may foster chronobiological homeostasis and ultimately health. To this end, it remains to identify zeitgebers comprehensively and to coordinate or align the information from multiple timecues as much as possible to reinforce each other with resulting high zeitgeber strength in appropriate time windows.

The 2017 Nobel Prize in Physiology or Medicine instilled additional interest in the field of chronobiology. Sport, sports medicine, and exercise physiology may be particularly relevant fields in which chronobiology should be explored as exercise has been discussed as a potential zeitgeber for the human circadian system. ${ }^{18-22}$ Therefore, we explored the validity of that hypothesis through a systematic review of the literature.

\section{METHODS}

A systematic literature search of the PubMed database was conducted on 19 October 2017 for relevant experimental, field and epidemiological studies and reports concerning chronobiology and exercise/physical activity in humans. We combined various search terms pertaining to the hypothesised human circadian system and exercise-zeitgeber relationship in addition to performance and health (table 1) and filtered the returned studies by title, abstract and main text content and findings (figure 1). We further searched the bibliographies of chronobiology and exercise relevant literature and supplemented the returned literature where appropriate (figure 1). Inclusion and exclusion criteria are presented in table 1. Data extraction and interpretation was initially performed by PL. Interpretation of data was also provided by coauthors. Potential sources of bias in the extracted studies are discussed as our own interpretation. Synthesis of studies involved categorisation by type of zeitgeber evidence and outcome measurements (described in the following paragraphs).

To delineate exercise as a zeitgeber for humans, there are a number of criteria it must be able to fulfil as originally put forward by Aschoff in the 1950's ${ }^{2}$ and still relevant today: (i) If the zeitgeber is switched off,

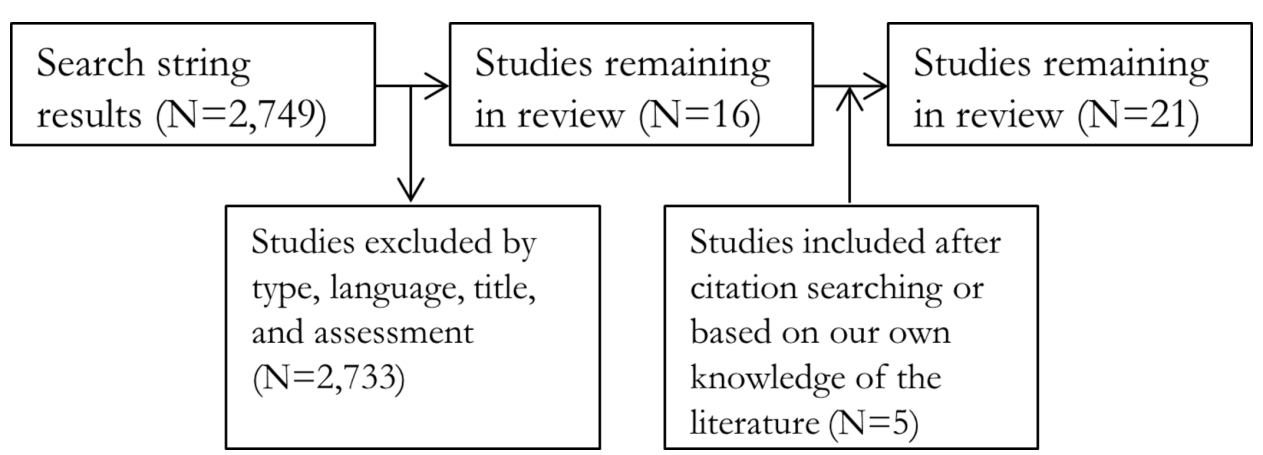

Figure 1 Preferred Reporting Items for Systematic Reviews and Meta-Analyses (PRISMA) flow diagram. 
the biological periodicity-if sustained-should eventually begin to deviate from the precise 24-hour period duration; (ii) reversing a continuously periodic zeitgeber or regular changes of conditions, such as the change of light and dark, must lead to an inversion of the biological periodicity; moreover, temporal shifts of the zeitgeber must lead to phase-shifts against the external time; (iii) depending on the zeitgeber effectiveness, changes of the biological periodicity can be expected by increasing or decreasing zeitgeber frequency. We shall apply these criteria in our synthesis. Furthermore, there are confounders and effect modifiers that we need to be aware of. For instance, when addressing the effectiveness of zeitgebers, Aschoff emphasised that-in most settings—several zeitgebers compete. Moreover, within any given zeitgeber multiplicity, entrainment is determined by both external intensity and internal susceptibility. ${ }^{2}$ Determinants of the strength of zeitgebers include intensity, duration, phase timing and periodic frequency while the susceptibility of the receiving organism to such information can be assessed by the phase of internal time (indicating a phase response curve),${ }^{23}$ and potentially by circadian system robustness. ${ }^{24}$ In effect, multiple zeitgebers such as light, noise, meals or social contacts may act synergistically or antagonistically, that is, they interact in terms of their 'push' or 'pull' on circadian rhythm phase. ${ }^{2}$ Best evidence supporting the hypothesis would include an entraining ability of the exercise zeitgeber with circadian phase drifting after removal of that zeitgeber. Additional support for the hypothesis would include evidence for an exercise phase response curve in phaseshifting a biological rhythm. Time-of-day dependent effects of exercise, which support the existence of a phase response curve, will also be discussed as potential evidence.

Overall, support for the 'exercise is a zeitgeber for humans' hypothesis should include a sufficiently strong and timed exercise stimulus being able to phase-shift the circadian rhythm of the SCN in accordance with Aschoff's blueprint. ${ }^{2}$ If there are no data to this effect, other biological processes co-governed with circadian rhythmicity must be assessed as proxies for SCN output for example, body temperature (BT), melatonin rhythm, exercise performance rhythm or sleep rhythm. Relevant chronotype information, that is, cases wherein it may be used as a proxy for circadian phase, will also be considered. While proxies are not without limitations, ${ }^{25}$ they are frequently necessary in human circadian biology research. Diminishing effects on rhythm amplitudes may result from an exercise stimulus representing conflicting zeitgeber information or differential speeds of rhythms entraining to the exercise stimulus phase and period. Additionally, the zeitgeber may or may not affect individual rhythm amplitudes. A 'washout' period may be required to delineate specific effects on the circadian system from more immediate 'masking' effects of exercise. These criteria are also reviewed in detail elsewhere. ${ }^{26}$

\section{RESULTS}

The systematic literature search returned 2749 articles. We determined 16 relevant for our synthesis based on the inclusion and exclusion criteria. A further five articles were added based on citation searching and our knowledge of the field (figure 1).

There are no studies reporting effects of exercise as a zeitgeber (or indeed otherwise) on the human SCN. This is expected given the difficulty in accessing the human SCN. Furthermore, there are no reports of chronotype changes following exercise intervention. Evidence for a phase response curve to exercise in humans has been reviewed by Edwards $e t a l^{26}$ and our systematic search revealed no new studies that have attempted to demonstrate a phase response curve to exercise in humans. Edwards et alnote that these studies represent supporting evidence that exercise might be a human zeitgeber but fall short of conclusively claiming that an exercise-zeitgeber has been demonstrated for humans. ${ }^{26}$ Thus, evidence for phase response curves will only be considered in brief.

There is abundant evidence of phase-shifting effects of exercise on commonly measured proxies of circadian rhythm (table 2). Exercise, depending on time of day or internal time of stimulus application, has been shown to phase-shift the melatonin, thyroid stimulating hormone (TSH) and BT rhythms. ${ }^{19} 27-32$ However, whether this effect is the result of masking or whether it persists after stimulus removal is unclear. From one study, no significant effect from the first exercise stimulus was observed, rather periodic bouts were required to manifest melatonin phase changes. ${ }^{19}$ This would suggest a zeitgeber effect rather than a masking effect. ${ }^{19}$ Buxton et al observed counter-intuitive phase-shifting responses to exercise at certain times with morning exercise eliciting phase delays and evening exercise eliciting phase advances. ${ }^{27}$ The authors plotted a tentative phase response curve based on their data. ${ }^{27}$ That the responsiveness to exercise followed a different timing pattern than would be expected for light may suggest the phaseshifting was the result of exercise per se and not light exposure. ${ }^{26}$ Van Reeth et al also plotted a tentative phase response curve to exercise. ${ }^{30}$ In a shortened forced sleepwake schedule study, exercise did not aid adaptation to the new schedule, rather the melatonin ascending limb was phase-delayed compared with non-exercise by day $8 .^{31}$ Furthermore, the phase points of the rhythm shifted in a temporally different manner with the descending phase not statistically different between groups after the initial 8 days but different after 14 days. ${ }^{31}$ In other words, some phase-shift differences took longer to manifest than others. If the effects observed are immediate effects rather than entraining effects, the time-period for manifestation of phase-shifts suggests an adaptation to the immediate effects. We would find this more difficult to reconcile than entrainment. In a longitudinal case study, high intensity exercise delayed the expression of clock genes in human hair cells compared with a following period of no exercise. ${ }^{33}$ Unfortunately, the 
Table 2 Exercise can phase-shift melatonin, thyroid stimulating hormone (TSH) and body temperature (BT)

\begin{tabular}{|c|c|}
\hline Author, year & Finding \\
\hline Van Reeth et al $1994^{30}$ & $\begin{array}{l}\text { A 3-hour sport stimulus placed around the time of the minimum core BT phase delayed both } \\
\text { melatonin and TSH rhythms. The stimulus timing was related to magnitude of phase-shifting effects. }\end{array}$ \\
\hline Miyazaki et al $2001^{31}$ & $\begin{array}{l}\text { Exercise phase-shifts the melatonin rhythm in a shortened forced sleep-wake routine different from } \\
\text { controls. }\end{array}$ \\
\hline Edwards et al $2002^{32}$ & $\begin{array}{l}\text { Exercise performed at specific times can phase-delay or phase-advance the core body temperature } \\
\text { rhythm. }\end{array}$ \\
\hline Baehr et al $2003^{28}$ & $\begin{array}{l}\text { Exercise at the beginning of habitual sleep time can phase-shift the melatonin rhythm more-so than } \\
\text { controls (awake). }\end{array}$ \\
\hline Buxton et al $2003^{27}$ & Exercise stimuli at a specific internal time can phase-shift the melatonin rhythm. \\
\hline Barger et al $2004^{19}$ & $\begin{array}{l}\text { Exercise in dim light can phase-delay dim light melatonin onset in constant routine with magnitude } \\
\text { related to stimulus timing. }\end{array}$ \\
\hline Okamoto et al $2013^{33}$ & Habitual nightly sport can phase-delay hair cell clock gene expression. \\
\hline Youngstedt et al $2016^{29}$ & $\begin{array}{l}90 \text { mins exercise on night } 2 \text { of a 3-hour ultra short sleep-wake cycle or } 90 \text { mins of light followed by } \\
\text { exercise } 4.5 \text { hours later can phase-delay the melatonin rhythm as much as a light stimulus alone and } \\
\text { additively, respectively. }\end{array}$ \\
\hline
\end{tabular}

first non-exercise circadian measurements were taken $\sim 3$ weeks after the exercise period ended; thus, a drifting of the phase was not tested. ${ }^{33}$ Added to this, phase differences were observed between the initial exercise-period circadian measurements and the second exercise-period circadian measurements. ${ }^{33}$ Hence, it is difficult to conclude that exercise was the zeitgeber. For instance, seasonal effects on phase of entrainment might have been detected. A repeat of this study, with more immediate observations after removal of the exercise stimulus could provide interpretable evidence of an exercise-induced circadian stimulus.

Several studies demonstrate that habitual external timing of exercise can (in a time-dependent manner) differentially affect the diurnal variation in performance (table 3). ${ }^{34-39}$ These studies are longitudinal involving repeated measures testing with several groups exercising at different times per day. However, we do not know how the different exercise schedules may have affected the timing of other zeitgeber exposure. For instance, morning exercise groups, as opposed to evening exercise groups, may be waking up earlier and being exposed to light earlier in the day. Over several weeks, they will entrain to this earlier light exposure, and their performance rhythm will also be expected to advance, which could explain the observations. On the other hand, some studies noted no differences in morning or evening BT between morning-trained and evening-trained groups which suggests either light exposures are not different between groups or are not sufficiently different to differentially affect the BT rhythm. ${ }^{34}$ A case study of exercise earlier in the day, measuring BT at several time points, identified changes in BT amplitude post-intervention but not in the observed phase timing of peaks or troughs. ${ }^{38}$ Another study demonstrates changes in diurnal variation of performance but not in the diurnal rhythm of testosterone or cortisol. ${ }^{39}$ This leads to the question: "why might the performance rhythm phase-shift separately

Table 3 Habitual time of exercise and diurnal variation in performance

\begin{tabular}{|c|c|}
\hline Author, year & Finding \\
\hline Souissi et al $2002^{37}$ & $\begin{array}{l}\text { The time-of-day of exercise ( } 6 \text { weeks) affects diurnal variation in muscular strength and anaerobic } \\
\text { performance, measured } 2 \text { weeks postintervention. }\end{array}$ \\
\hline Blonc et al $2010^{40}$ & The time-of-day of exercise (5 weeks) did not affect diurnal variation in muscular strength. \\
\hline Chtourou et al $2012^{35}$ & The time-of-day of exercise (12 weeks) affects diurnal variation in muscular strength. \\
\hline Imafuku $2016^{38}$ & Exercise affected BT amplitude but not phase. \\
\hline Kuusmaa et al $2016^{39}$ & $\begin{array}{l}\text { Exercise-type sequence and time-of-day (12-24 weeks) affects diurnal variations in performance after } \\
12 \text { weeks but not cortisol or testosterone. }\end{array}$ \\
\hline Zbidi et al $2016^{34}$ & $\begin{array}{l}\text { The time-of-day of exercise ( } 6 \text { weeks) affects diurnal variation in muscular strength but not BT, } \\
\text { measured from } 48 \text { hours postintervention. }\end{array}$ \\
\hline
\end{tabular}


Table 4 Exercise interaction with the circadian system to improve health

\begin{tabular}{ll}
\hline Author, year & Finding \\
\hline Van Someren et al $1997^{44}$ & $\begin{array}{l}\text { Long-term fitness training improves the circadian rest-activity rhythm in healthy elderly males, } \\
\text { measured 1 month postintervention. }\end{array}$ \\
\hline Montaruli et al 200946 & $\begin{array}{l}\text { The time-of-day of exercise was associated with the circadian rhythm and sleep response to } \\
\text { transmeridian travel (although chronotype and light exposure may have played a role). }\end{array}$ \\
\hline Fairbrother et al $2014^{45}$ & $\begin{array}{l}\text { Exercise, depending on time of day, could differentially augment nocturnal blood pressure dips, } \\
\text { time spent in different sleep-stages, and sleep-onset latencies in the 24 hours following the } \\
\text { stimulus. }\end{array}$ \\
Chen et al $2015^{42}$ & $\begin{array}{l}\text { Circadian rhythm is associated with physical activity and objective sleep in patients with lung } \\
\text { cancer. }\end{array}$ \\
Chen et al $2016^{43}$ & Exercise improves circadian rest-activity rhythm in patients with lung cancer.
\end{tabular}

from other well-described circadian rhythms such as BT, cortisol or testosterone?" A 'masking effect' of exercise on exercise performance could offer an explanation. However, this non-circadian effect needs to have lasted from 48 hours up to 2 weeks after the last exercise session as per the timing of the post-intervention testing session in these studies. ${ }^{34} 3637$ What 'washout' period would be required to observe circadian effects from masking effects remains open. One study indicated no effect of habitual time of exercise on diurnal variation in performance. ${ }^{40}$ Different to the other studies reported here,${ }^{34-39}$ the authors did not observe a diurnal variation in performance to begin with. ${ }^{40}$ They suggest that the tropical environmental conditions in Guadeloupe may have played a role. ${ }^{40}$ Remarkably, even an acute bout of submaximal cycling performed the day before a timetrial improved performance when it occurred at the same time-of-day as the time-trial. ${ }^{41}$ The authors suggest chronotype preference, fatigue (glycogen and lactate levels), circadian phase-shift and time familiarisation as the potential explanations, but that the latter is the most likely. ${ }^{41}$ However, other studies have observed phaseshifting of performance independent of $\mathrm{BT}^{34} 36$; thus, a circadian phase-shift of performance rhythm independent of BT may be possible.

The following studies demonstrate the potential for exercise to interact with the human circadian system to improve health (table 4). Chen et al, after detecting associations between poor rest-activity rhythms and poor sleep in patients with lung cancer, ${ }^{42}$ found that 6 months of prescribed individual-specific exercise improved objective sleep in patients with lung cancer compared with a "usual care" control group. ${ }^{43}$ The effect was strongest in individuals with poorer rest-activity rhythms. ${ }^{43}$ That the exercise targets were specific to each patient based on activity scores at the beginning of the trial could, at least in part, control for general fitness improvements affecting sleep. Thus, that this effect was observed more strongly in patients with poorer rest-activity rhythms indicates a potential circadian component to the exercise effect. Although time of day of exercise was not noted, 3 months of exercise improved intradaily rest-activity rhythmicity measured 1 month postintervention in an elderly population. ${ }^{44}$ The participants had discontinued the exercise regimen in this post 1 month period. ${ }^{44}$ Moreover, the effects were not attributed to changes in light exposure as no participants increased outdoor activities. ${ }^{44}$ There were no fitness and pre-exercise rest-activity rhythm associations, thus, improved fitness is likely to play only a minor role, if any, in the observed improved rest-activity rhythms. Exercise, depending on time of day, could differentially augment nocturnal blood pressure "dips", time spent in different sleep-stages and sleeponset latencies in the 24 hours following the stimulus. ${ }^{45}$ Consecutive measurements or measurements following a longer "washout" period were not taken. Thus, potential zeitgeber effects cannot be distinguished from masking effects. One month of evening exercise in Milan, prior to travel to New York, proved better than 1 month of morning exercise in terms of improving sleep and the circadian rhythm of rest-activity post flight. ${ }^{46}$ The authors report that the time-of-day of sport contributed to the circadian rhythm and sleep response to transmeridian travel. ${ }^{46}$ The authors state that bedtimes and waking times were set for each subject in this study, but they were not reported in the manuscript. ${ }^{46}$ Thus, both circadian phase and daily light exposure could have significantly contributed to faster adaptation to sleeping at the prescribed bedtime in New York in the evening-trained group.

\section{DISCUSSION}

Clearly, exercise has circadian rhythm phase-shifting properties, both immediate as observed in assessments of classic circadian hormone and BT rhythms and more long-term as observed by studies on the timing of peak performance. Regarding health and disease, informed timing of exercise may be useful as an adjunct therapeutic or preventative strategy to foster chronobiological homeostasis. Equally clearly, when taken together, these studies strongly suggest that exercise is a zeitgeber signal for the human circadian system.

Regarding Aschoff's criterion 1, no studies wherein a potential periodic sport zeitgeber is switched off and a drift of circadian phase back to its original timing were returned by our systematic search. However, several studies took measurements of the diurnal variation in 
performance from 48 hours up to several weeks after the exercise intervention is removed and found that the change in diurnal variation persisted. ${ }^{34} 3637$ On the one hand, these measurements are not strictly indicative of a performance circadian rhythm. On the other hand, it is difficult to reconcile why these effects persist if not for the circadian system. We put forward that such studies taking repeated circadian measurements following a 'switched off' exercise zeitgeber may be feasible in athletes who are injured and unable to participate in periodic sport. In contrast to being switched off, the switching on of an exercise-zeitgeber in humans is, in effect, presented in the literature. In contrast to switching off, we should expect that switching on causes a given biological periodicity or entrained rhythm to deviate. Indeed, we observe this in the phase-shifting effects of exercise on melatonin, BT, and TSH. ${ }^{19}{ }^{27-32}$ In many cases, recurrent zeitgeber signalling is required before a significant phase-shift is observed. Evidence of criterion 2 is also compatible with exercise being a zeitgeber insofar as there is evidence that the timing of exercise is crucial to eliciting phase-shifting effects and differentiating between phase-delaying and phase-advancing effects. ${ }^{19}$ 26-32 In regard to Aschoff's zeitgeber criterion 3 , there is one study where the zeitgeber frequency is increased alongside a shortened sleep schedule; namely periodic recurrence every 23 hours and 40 mins. ${ }^{31}$ Contrary to the potential zeitgeber aiding entrainment to the new period, exercise phase-delayed the melatonin rhythm in comparison to controls. ${ }^{31}$

The zeitgeber effects of light depend on frequency, timing, intensity and light history. We have discussed facets of frequency and timing of exercise when applying Aschoff's zeitgeber criteria above. We lack detailed and comparable studies of varying zeitgeber effects of different exercise intensities and exercise history. Regarding the latter, exercise has been shown to elicit greater improvements in the rhythmicity of individuals with poor circadian rhythm ${ }^{434}$ conveying the notion that the history of rhythmic activity is important. Regarding the former, we can make inferences based on the fact that the experimental groups of the studies will not have been completely inactive during the study (ie, that some activity beyond the stimulus will be necessary for daily life, eg, walking, driving, cooking, cleaning, work etc). In this regard, the exercise stimulus represents a higher intensity of physical activity compared with other activities. Following this, the higher activity intensity affected phase-shifting responses. This may also be considered as supporting evidence for Aschoff's criterion 2.

Interestingly, two studies demonstrate counter-intuitive phase-shifting effects due to exercise that is, different directions to light. ${ }^{27} 31$ Moreover, changes in performance rhythms have been observed independent of other rhythms. ${ }^{39}$ This opens questions of whether phaseshifting effects of exercise are acting at the level of the $\mathrm{SCN}$, and whether central output determining performance rhythms is truly from the SCN. Javierre et al have already speculated that physical performance rhythm may be co-governed by a different oscillator to that of $\mathrm{BT}^{47}$

Concerning limitations of the synthesised studies, the low frequency measurements over the course of a day in the diurnal variation in performance studies and often low numbers of participants (as can be the case in human interventions studies) are apparent. We also note a limitation in terms of potential for selection bias. Studies into exercise effects on humans, especially at certain times of day, may attract participants whose own schedules suit the schedules used in these studies. Thus, this may lend to internal time and chronotype bias among participants, although chronotype is often accounted for. The authors note that only one study observed no phase-shifting effects of an exercise stimulus; the potential exists that more studies demonstrating no zeitgeber effects remain unpublished due to publication bias. Lastly, most studies in tables 2 and 3 consider effects on participants who have not regularly trained for several months and we lack comparison with elite athletes, sedentary individuals and individuals of different ages. Differences in physical fitness and age may affect sensitivity to an exercise stimulus.

Aschoff suggested that in most instances there are several zeitgebers, that they compete and that regularly one will take the lead and dominate over the others. ${ }^{2}$ This has been complemented by Crowley and Eastman ${ }^{48}$ and with specific regard to sport by Youngstedt $e t a l^{29}$ showing that rather than one zeitgeber dominating a zeitgeber competition, in many settings there will be zeitgeber interaction ${ }^{29} 48$-be it antagonistic or synergistic. In this vein, we suspect that in some individuals who actively engage in exercise, the zeitgeber effects of exercise may compete-or act antagonistically—against other zeitgebers to the detriment of performance and of chronobiological health. In effect, this may, of course, be less conspicuous due to the known benefits of exercise per se. However, identification and removal of potential adverse zeitgeber effects, that is, appropriate alignment of exercise within the zeitgeber multiplicity and in reference to internal time, may provide athletes with added effects of exercise and/or provide a 'difference-making' effect on performance. Importantly, in our view, having unambiguous and strong-rather than confusing and weak-zeitgeber information at the right times could foster chronobiological homeostasis and ultimately health and act as a performance enhancer.

In closing, we do not expect that our systematic literature search is factually complete. Indeed, we do expect that some research targeting the nexus of chronobiology and performance will have been conducted in secrecy to allow competitive edges and thus remain unpublished. The answer to the question as to whether exercise has been conclusively demonstrated to be a zeitgeber for humans in a single study is, as of yet, no-but the supporting evidence of an exercise-zeitgeber for humans is nonetheless strong. 
For health specialists and sport performance specialists, sufficient evidence thus far supports a role for exercise as a zeitgeber for the human circadian system and for chronobiological insights affecting performance that may be used to foster health and improve performance. Exercise, appropriately timed, must be advocated in line with 'zeitgeber hygiene' and preventive medicine against circadian disruption. Indeed, when not contraindicated as judged by physicians, exercise as a zeitgeber to foster chronobiological health would be preferable to pharmacomedication due to its additional known beneficial effects on health. For the purist scientist, the question of 'how' can exercise be a zeitgeber remains unanswered. We suspect it likely that the exercise stimulus modifies the internal environment in a manner that allows systemic signalling to the circadian coordinating centres of the brain (eg, myokine circadian rhythm ${ }^{49}$ ). In the evolutionary context, the ability to shift the timing of peak performance or sleep based on previous timing of an activity stimulus can be envisaged to have provided competitive edges (viz., predictive homeostasis) to organisms.

In conclusion, informed timing of exercise can be advocated in performance and disease contexts as a physical enhancer and as an adjunct therapeutic or preventative strategy.

Contributors LK, JVG, TCE: began initial work into this area. PL, TCE: designed the current study. PL: carried out the literature search and synthesis. TCE, HK: reviewed historical German language publications relevant to zeitgebers. PL, TCE: drafted the initial manuscript. All authors provided important intellectual content and approved the final version of the manuscript.

Funding The authors have not declared a specific grant for this research from any funding agency in the public, commercial or not-for-profit sectors.

Competing interests None declared.

Patient consent Not required.

Provenance and peer review Not commissioned; internally peer reviewed.

Data sharing statement There is no additional data.

Open access This is an open access article distributed in accordance with the Creative Commons Attribution Non Commercial (CC BY-NC 4.0) license, which permits others to distribute, remix, adapt, build upon this work non-commercially, and license their derivative works on different terms, provided the original work is properly cited, appropriate credit is given, any changes made indicated, and the use is non-commercial. See: http://creativecommons.org/licenses/by-nc/4.0/

\section{REFERENCES}

1. Aschoff J. Die 24-Stunden-Periodik der Maus unter konstanten Umgebungsbedingungen. Naturwissenschaften 1951;38:506-7.

2. Aschoff J. Zeitgeber der tierischen Tagesperiodik. Naturwissenschaften 1954;41:49-56.

3. Halberg F. [Physiologic 24-hour periodicity; general and procedural considerations with reference to the adrenal cycle]. Int $Z$ Vitaminforsch Beih 1959;10:225-96.

4. Hattar S, Liao HW, Takao M, et al. Melanopsin-containing retinal ganglion cells: architecture, projections, and intrinsic photosensitivity. Science 2002;295:1065-70.

5. Erren TC, Reiter RJ. Melatonin: a universal time messenger. Neuro Endocrinol Lett 2015;36:187-92.

6. Kalsbeek A, Palm IF, La Fleur SE, et al. SCN outputs and the hypothalamic balance of life. J Biol Rhythms 2006;21:458-69.

7. Gery S, Koeffler HP. The role of circadian regulation in cancer. Cold Spring Harb Symp Quant Biol 2007;72:459-64.

8. Vetter C, Fischer D, Matera JL, et al. Aligning work and circadian time in shift workers improves sleep and reduces circadian disruption. Curr Biol 2015;25:907-11.
9. Lewis P, Foster RG, Erren TC. Ticking time bomb? High time for chronobiological research. EMBO Rep 2018;19:e46073.

10. IARC Working Group on the Evaluation of Carcinogenic Risks to Humans. Painting, firefighting, and shiftwork. IARC Monogr Eval Carcinog Risks Hum 2010;98:9-764.

11. Czeisler CA. Perspective: casting light on sleep deficiency. Nature 2013;497:S13.

12. Stevens RG, Blask DE, Brainard GC, et al. Meeting report: the role of environmental lighting and circadian disruption in cancer and other diseases. Environ Health Perspect 2007;115:1357-62.

13. Stevens RG, Davis S, Thomas DB, et al. Electric power, pineal function, and the risk of breast cancer. Faseb $J$ 1992;6:853-60.

14. Kerenyi NA, Pandula E, Feuer G. Why the incidence of cancer is increasing: the role of 'light pollution'. Med Hypotheses 1990;33:75-8.

15. Reiter RJ, Tan DX, Madrid JA, et al. When the circadian clock becomes a ticking time bomb. Chronobiol Int 2012;29:1286-7.

16. West AC, Smith L, Ray DW, et al. Misalignment with the external light environment drives metabolic and cardiac dysfunction. Nat Commun 2017;8:417

17. Czeisler CA. Duration, timing and quality of sleep are each vital for health, performance and safety. Sleep Health 2015;1:5-8.

18. Chtourou $\mathrm{H}$, Souissi $\mathrm{N}$. The effect of training at a specific time of day: a review. J Strength Cond Res 2012;26:1984-2005.

19. Barger LK, Wright KP, Hughes RJ, et al. Daily exercise facilitates phase delays of circadian melatonin rhythm in very dim light. Am J Physiol Regul Integr Comp Physiol 2004;286:R1077-84.

20. Hamaguchi $Y$, Tahara $Y$, Hitosugi M, et al. Impairment of Circadian Rhythms in Peripheral Clocks by Constant Light Is Partially Reversed by Scheduled Feeding or Exercise. J Biol Rhythms 2015;30:533-42.

21. Eastman $\mathrm{Cl}$, Hoese EK, Youngstedt $\mathrm{SD}$, et al. Phase-shifting human circadian rhythms with exercise during the night shift. Physiol Behav 1995;58:1287-91.

22. Ammar A, Chtourou $\mathrm{H}$, Souissi N. Effect of Time-of-Day on Biochemical Markers in Response to Physical Exercise. J Strength Cond Res 2017;31:272-82.

23. Hastings JW, Sweeney BM. A persistent diurnal rhythm of luminescence in gonyaulax polyedra. Biol Bull 1958;115:440-58.

24. Lewis $P$, Erren TC. Perinatal light imprinting of circadian clocks and systems (PLICCS): A signature of photoperiod around birth on circadian system stability and association with cancer. Chronobiol Int 2017;34:782-801.

25. Zeitzer JM. When is a proxy not a proxy? The foibles of studying non-image forming light. J Physiol 2018;596:2029-30.

26. Edwards BJ, Reilly T, Waterhouse J. Zeitgeber -effects of exercise on human circadian rhythms: what are alternative approaches to investigating the existence of a phase-response curve to exercise? Biol Rhythm Res 2009;40:53-69.

27. Buxton OM, Lee CW, L'Hermite-Baleriaux M, et al. Exercise elicits phase shifts and acute alterations of melatonin that vary with circadian phase. Am J Physiol Regul Integr Comp Physiol 2003:284:R714-24.

28. Baehr EK, Eastman Cl, Revelle W, et al. Circadian phase-shifting effects of nocturnal exercise in older compared with young adults. Am J Physiol Regul Integr Comp Physiol 2003;284:R1542-50.

29. Youngstedt SD, Kline CE, Elliott JA, et al. Circadian Phase-Shifting Effects of Bright Light, Exercise, and Bright Light + Exercise. J Circadian Rhythms 2016;14:2

30. Van Reeth O, Sturis J, Byrne MM, et al. Nocturnal exercise phase delays circadian rhythms of melatonin and thyrotropin secretion in normal men. Am J Physiol 1994;266(6 Pt 1):E964-74.

31. Miyazaki T, Hashimoto S, Masubuchi S, et al. Phase-advance shifts of human circadian pacemaker are accelerated by daytime physical exercise. Am J Physiol Regul Integr Comp Physiol 2001:281:R197-205.

32. Edwards B, Waterhouse J, Atkinson G, et al. Exercise does not necessarily influence the phase of the circadian rhythm in temperature in healthy humans. J Sports Sci 2002;20:725-32.

33. Okamoto A, Yamamoto T, Matsumura R, et al. An out-of-lab trial: a case example for the effect of intensive exercise on rhythms of human clock gene expression. J Circadian Rhythms 2013;11:10.

34. Zbidi S, Zinoubi B, Vandewalle H, et al. Diurnal Rhythm of Muscular Strength Depends on Temporal Specificity of Self-Resistance Training. J Strength Cond Res 2016;30:717-24.

35. Chtourou $\mathrm{H}$, Chaouachi $\mathrm{A}$, Driss $\mathrm{T}$, et al. The effect of training at the same time of day and tapering period on the diurnal variation of short exercise performances. J Strength Cond Res 2012;26:697-708.

36. Chtourou H, Driss T, Souissi S, et al. The effect of strength training at the same time of the day on the diurnal fluctuations of muscular anaerobic performances. J Strength Cond Res 2012;26:217-25. 
37. Souissi N, Gauthier A, Sesboüé B, et al. Effects of regular training at the same time of day on diurnal fluctuations in muscular performance. J Sports Sci 2002;20:929-37.

38. Imafuku M. Body temperature rhythm and control of the time of the best physical condition by performing physical labor. Chronobiol Int 2016;33:431-4.

39. Küüsmaa M, Schumann M, Sedliak M, et al. Effects of morning versus evening combined strength and endurance training on physical performance, muscle hypertrophy, and serum hormone concentrations. Appl Physiol Nutr Metab 2016;41:1285-94.

40. Blonc S, Perrot S, Racinais S, et al. Effects of 5 weeks of training at the same time of day on the diurnal variations of maximal muscle power performance. J Strength Cond Res 2010;24:23-9.

41. Edwards BJ, Edwards W, Waterhouse J, et al. Can cycling performance in an early morning. laboratory-based cycle time-trial be improved by morning exercise the day before? Int J Sports Med 2005;26:651-6.

42. Chen HM, Wu YC, Tsai CM, et al. Relationships of Circadian Rhythms and Physical Activity With Objective Sleep Parameters in Lung Cancer Patients. Cancer Nurs 2015;38:215-23.
43. Chen HM, Tsai CM, Wu YC, et al. Effect of walking on circadian rhythms and sleep quality of patients with lung cancer: a randomised controlled trial. Br J Cancer 2016;115:1304-12.

44. Van Someren EJ, Lijzenga C, Mirmiran M, et al. Long-term fitness training improves the circadian rest-activity rhythm in healthy elderly males. J Biol Rhythms 1997;12:146-56.

45. Fairbrother K, Cartner B, Alley JR, et al. Effects of exercise timing on sleep architecture and nocturnal blood pressure in prehypertensives. Vasc Health Risk Manag 2014;10:691-8.

46. Montaruli A, Roveda E, Calogiuri G, et al. The sportsman readjustment after transcontinental flight: a study on marathon runners. J Sports Med Phys Fitness 2009;49:372-81.

47. Javierre $\mathrm{C}$, Ventura JL, Segura $\mathrm{R}$, et al. Is physical training a good synchronizer of the performance? Rev Esp Fisiol 1997;53:239-45.

48. Crowley SJ, Eastman $\mathrm{Cl}$. Phase advancing human circadian rhythms with morning bright light, afternoon melatonin, and gradually shifted sleep: can we reduce morning bright-light duration? Sleep Med 2015;16:288-97.

49. Perrin L, Loizides-Mangold U, Skarupelova S, et al. Human skeletal myotubes display a cell-autonomous circadian clock implicated in basal myokine secretion. Mol Metab 2015;4:834-45. 